Institute for Fundamental Theory Preprint UFIFT -HEP-91-12

\title{
Supermatrix Models
}

\author{
ScotT A. Yost ${ }^{\star}$ \\ Department of Physics
}

University of Florida, Gainesville, FL 32611

\begin{abstract}
Random matrix models based on an integral over supermatrices are proposed as a natural extension of bosonic matrix models. The subtle nature of superspace integration allows these models to have very different properties from the anajogous bosonic models. Two choices of integration slice are investigated. One leads to a perturbative structure which is reminiscent of, and perbaps identical to, the usual Hermitian matrix models. Another leads to an eigenvalue reduction which can be described by a two cumponent plasma in one dimension. A stationary point of the model is described.
\end{abstract}

* Work supported in part by the Department of Energy, contract DE-FG05-86ER-40272. Address after September 1, 1991: Department of Physics and Astronomy, University of Tennesser, Knoxville, TN 37996. 


\section{Introduction}

Integrals over random matrices have had a variety of physical and mathematical applications. They were originally proposed as a statistical model for studying the distribution of energy levels of highly excited states of nuclei[1-3]. The matrices are taken to be from some ensemble of diagonalizable matrices, usually Hermitian or unitary. The perturbative expansion of the free energy was found to generate Feynman rules which are useful in solving numerous graph-counting problems[4-6]. More recently, these properties have been found to make certain scaling limits of matrix models useful for studying two-dimensional gravity, or string theory in "less than one dimension[7-11]."

It is natural to consider the extension of these models to supermatrices, which represent the linear transformations of a vector space having both even (bosonic) and odd (fermicnic) coordinates. All of the algebraic and analytic methods needed to define ordinary matrix models have extensions to supermatrices. Moreover, supermatrices come in two types: $c$-type, which preserve the coordinate type of a vector, and a-type, which interchange fermions and bosons. This suggests that a model which includes both could be useful in defining subcritical superstrings.

This paper will concentrate on $c$-type matrix models, since these are the most closely analogous to the ordinary models. In particular, "almost all" $c$-type matrices are diagonalizable ( $a$-type ones need not be), and diagonalization is a powerful tool in rendering bosonic matrix models tractable. Unfortunately, the superunitary angular integral generally does not decouple, so some nonperturbative methods, such as the orthogonal polynomial method, will lose their power.

Since a $c$-type supermatrix can be decomposed into a two by two block matrix, with bosonic matrices on the diagonal and fermionic ones off it, one may suspect that they already contain enough ingredients to define a discretized superstring

† A general reference, and the source of all terminology and conventions used here, is ref. [12]. 
model. However, this seems unlikely to be a proper interpretation, since the potential contains only bosonic coupling constants, and since the eigenvalues are all bosonic. If one thinks of $c<1$ strings as integrable systems[13], what is wanted is a superintegrable system[14], which requires a model with fermionic coupling constants tc generate both even and odd $K d V$-type flows.

One of the greatest distinctions between supermatrix models and ordinary ones is that superspace integration is inherently ambiguous. There is no geometrically natural reason to restrict the bosonic components of the supermatrix to be pure real or complex numbers. In general, they are simply even elements (c-numbers) in a Grassmann algebra, consisting of a body which is an ordinary number, and a soul made of even products of anticommuting numbers[12]. To define the integration domain requires specifying a slice through soul-space, and different choices really should be thought of as different models, with different properties.

Section 2 of this paper describes the general properties of supermatrix models. Section 3 describes the case when the even entries in the matrix are ordinary numbers. This choice gives the simplest perturbative expansion, which turns out to be very similar (perhaps identical) to that of an ordinary bosonic model. Section 4 describes integrals over matrices which are constrained to have pure real eigenvalues with no soul. (These are called physical supermatrices[12].) When gauge-fixed, such a model can be described by a physical system analogous to the Dyson gas of bosonic models. In this case, the physical analog is found to be a two-component plasma in one dimension. Although the technology for solving such a inatrix model is presently limited, a saddle point evaluation based on interacting dipoles is used to illustrate some features of the model.

Three very recent supermatrix model references may be of interest. A paper by C. Vaz[15] analyzes a special case of the antisymmetric supermatrix model, and one by L. A.lvarez-Gaumé and J. Manẽs compares the Hermitian matrix and supermatrix models. Finally, G. Gilbert and M.J. Perry[16] propose a quenched 
$c$-type supermatrix model which appears to incorporate genuine supersymmetry.

\section{Supermatrix Integration}

Supermatrix models can be defined by taking any bosonic mode! and replacing all of the ingredients by their superspace analogs. In particular, consider a Hermitian $c$-type supermatrix[12] (the only type to be used in this paper)

$$
M_{i j}=\left(\begin{array}{cc}
A_{\mu \nu} & B_{\mu \beta} \\
B_{\alpha \nu}^{\dagger} & C_{\alpha \beta}
\end{array}\right)
$$

where $A$ and $C$ are bosonic and Hermitian, and $B$ is fermionic. The notation indicates that $i, j, \ldots$ denote a general index, while $\mu, \nu, \ldots$ are bosonic and $\alpha, \beta, \ldots$ are fermionic. If $(-1)^{i}$ is +1 for bosonic indices and -1 for fermionic ones, then the supertrace is defined to be

$$
\operatorname{str} M=\sum_{i}(-)^{i} M_{i i}=\operatorname{tr} A-\operatorname{tr} C .
$$

For an arbitrary potential $V(M)$, the random Hermitian supermatrix model is defined by the partition function

$$
Z_{m n}(\beta)=\int_{\mathcal{S}} d M \exp (-\beta \operatorname{str} V(M))
$$

where $M$ is an $(m \mid n)$ supermatrix acting on vectors with $m$ commuting and $n$ anticommuting components, and

$$
d M=\prod d A_{\mu \nu} d C_{\alpha \beta} d^{2} B_{\mu \beta} .
$$

The measures for $A$ and $C$ are the usual linear Hermitian measures. The integration domain $\mathcal{S}$ is chosen so that the bodies of $A$ and $C$ range over the ordinary 
Hermitian matices, but the souls, which can contain even products of $B$ components, will be specified later. Since the supertrace is indefinite, the integral (2.3) will not actually exist for polynomial potentials. However, this is a technical problem that can be avoided by considering superunitary matrices instead, or by multiplying the potential by $i$ and inserting convergence factors.

When $\mathcal{S}$ is chosen such that the Gaussian integral (quadratic potential) can be evaluated, more general potentials can be handled by a perturbative expansion. This is true in particular when $A$ and $C$ are taken to be ordinary soul-less Hermitian matrices, the case considered in section 3 . The present section will consider methods which may be applied to more general cases.

In the bosonic models, the most powerful methods rely on diagonalization to obtain an integral over eigenvalues alone, factoring out the degeneracy due to the unitary angular integration $[3,5]$. Therefore, it is of interest to see what may be gained by diagonalizing the s'permatrix. A $c$-type hermitian supermatrix can be diagonalized by a superunitary transformaticn, except in certain singular cases. The singular cases occur when one of the eigenvalues of $A$ has the same body as an eigenvalue of $C$. In that limit, a pair of eigenvectors become bodyless, and cannot be part of an orthonormal basis, so the diagonalization cannot be carried out. However, this occurs only at isolated points in the integral.

Assume now that

$$
\mathcal{S}=\mathcal{D}^{\mathcal{U}}=\left\{U^{\dagger} \mathcal{D} U \mid U \in \mathcal{U}\right\}
$$

where $\mathcal{U}$ is $U(m \mid n)$, with a possibly unusual choice of souls. The body of $\mathcal{D}$ is just the set of $(m \mid n)$ real diagonal matrices, and all off-diagonal elements vanish. Following the classic matrix-model method[3,5], the integrand of (2.3) may be multiplied and divided by

$$
\Delta^{-1}(M)=\int_{\mathcal{U}} d U \prod_{i>j} \delta^{2}\left(U M U^{\dagger} !_{: j} .\right.
$$

Changing variables $M \rightarrow M^{U}=U^{\dagger} M U$ and using the invariance of the measure 
and supertrace leads to

$$
Z_{m n}(\beta)=\int_{\mathcal{L}^{\prime}} d U \int_{\mathcal{S} U} d \Lambda \Delta(\Lambda) \exp (-\beta \operatorname{str} V(\Lambda))
$$

where $\Lambda$ is the diagonal matrix of eigenvalues $\lambda_{i}$ of $M$.

An important difference from the bosonic case is immediately clear. The integral over the supergroup does not factorize. The choice of souls for the eigenvalues depends on the group element $U$. If this were a one-dimensional integral, the choice of souls would not have mattered, up to a surface term. But for multiple integrals, the dependence is nontrivial. Also, the evaluation of (2.6) depends on $\mathcal{U}$, which is algebraically $U(m \mid n)$, but may have unusual soul geometry.

A case where (2.7) can be evaluated may be obtained by restricting the eigenvalues to be pure real numbers, and $\mathcal{U}$ to be the standard $U(m \mid n)$ with its Haar measure. Then the set $\mathcal{S}$ consists of what deWitt[12] calls the "physical supermatrices." This case will be the subject of section 4 . It has the drawback that the constraint of having real eigenvalues cannot easily be included in (2.3), so that even the Gaussian integral becomes complicated, and perturbative Feynman rules are difficult to develop.

A possible compromise would be to diagonalize only $A$ and $C$, by performing a superunitary transformation $U \times V \in U(m) \times U(n)$. Then $B \rightarrow U B V^{\dagger}$. The Jacobians for $A$ and $C$ are the usual Vandermonde determinants, while the Jacobian for $B$ is just 1 , so

$$
Z_{m n}(\beta)=\int \prod d a_{\mu} d c_{\alpha} d B_{\nu \beta} \prod_{\mu<\nu}\left(a_{\mu}-a_{\nu}\right)^{2} \prod_{\alpha<\beta}\left(c_{\alpha}-c_{\beta}\right)^{2} \exp (-\beta \operatorname{str} V(M))
$$

where $M$ is now defined with $A=\operatorname{diag}(a), C=\operatorname{diag}(c)$, and the volume of $U(m) \times U(n)$ has been divided out. The remaining integral over $B$ can be replaced

\footnotetext{
$\star$ See p. 7 of [12] for a proof.
} 
by an integral over a $U(m \mid n) / U(m) \times U(n)$ coset, if desired. It is not clear whether (2.8) is a useful supplement to (2.3). This question will not be persued here.

A simple example may be helpful to clarify the issues in this section. Consider the case $m=n=1$. Then $A$ and $C$ become real even supernumbers $a$ and $c$, while $B$ becomes a complex odd Grassmann number $\beta$. The eigenvalues of $M$ are

$$
\lambda=a+(a-c)^{-1} \beta \beta^{*}, \quad \mu=c+(a-c)^{-1} \beta \beta^{*},
$$

and $U M U^{\dagger}$ is diagonal when the superunitary matrix is

$$
U=\left(\begin{array}{cc}
1-\frac{1}{2} \alpha \alpha^{*} & \alpha \\
-\alpha^{*} & 1+\frac{1}{2} \alpha \alpha^{*}
\end{array}\right)
$$

with $\alpha=(a-c)^{-1} \beta$.

The partition function will now have the form

$$
\begin{aligned}
Z & =\int d a d c d \beta d \beta^{*} \exp (-\operatorname{str} V(M)) \\
& =\int d \alpha d \alpha^{*} \int d \lambda d \mu(\lambda-\mu)^{-2} \exp (-V(\lambda)+V(\mu))
\end{aligned}
$$

This is a special case of $(2.7)$ with $d U=d \alpha d \alpha^{*}$ and $\Delta(\lambda, \mu)=(\lambda-\mu)^{-2}$. The unimportant diagonal subgroup of $U(1 \mid 1)$ is omitted. Since no even parameters are needed in $U$, there is no ambiguity in expressing the group manifold $\mathcal{U}$ in this case. The supernumbers $a$ and $c$ can be decomposed into body and soul, which have the form

$$
\begin{aligned}
& a=a_{B}+a_{S}=a_{B}+f\left(a_{B}, c_{B}\right) \beta \beta^{*} \\
& c=c_{B}+c_{S}=c_{B}+g\left(a_{B}, c_{B}\right) \beta \beta^{*},
\end{aligned}
$$

where $a_{B}$ and $c_{B}$ range over the real numbers, and $f$ and $g$ are ordinary realvalued functions. This form is unique if the souls depend only on $\beta$ and $\beta^{*}$. 
The integral can be reduced to an integral over the bodies by including a Jacobian factor

$$
\int d a d c=\int_{-\infty}^{\infty} d a_{B} d c_{B}\left[1+\left(f_{, 1}+g_{, 2}\right) \beta \beta^{*}\right]
$$

The presence of such factors can make a direct evaluation of (2.3) complicated for large matrices, even for the simplest potentials. It is useful to choose $\mathcal{S}$ so that either (2.3) or (2.7) is as simple as possible. These two cases are the subject of the following sections.

\section{Ordinary Hermitian Supermatrix Model}

The partition function (2.3) can be expressed most simply when $A$ and $C$ are chosen to be ordinary soul-less Hermitian matrices. This case will be referred to as the "ordinary Hermitian supermatrix model". No troublesome Jacobian factors of the form (2.13) complicate the evaluation, so the Gaussian integral can be evaluated exactly. More complicated potentials can be handled perturbatively, by developing Feynman rules[5]. The identification of dense Feynman diagrams with Riemann surfaces is the reason for the relevance of a scaling limit of ordinary matrix models to two-dimensional gravity[7-9]. Therefore, it should be expected that the connection to gravity can be made most directly for the ordinary Hermitian supermatrix model.

Consider a quadratic potential $\frac{1}{2} \operatorname{str} M^{2}$. The Feynman rules and Wick's theorem can be derived as in the usual case, by calculating the expectation value of a source $\exp (\operatorname{str}(J M))$, and varying with respect to $J$ to obtain the expectation values needed for a perturbative expansion. The propagator is simply

$$
\left\langle M_{i j} M_{k l}^{*}\right\rangle=(-)^{j} \delta_{i k} \delta_{j l}
$$

Wick contractions must always be carried out after permuting the matrices so that they are adjacent, keeping track of the signs $(-)^{(i+j)(k+l)}$ introduced by 


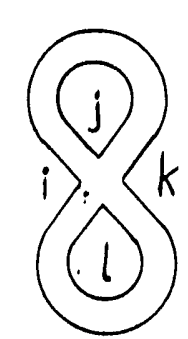

(a)

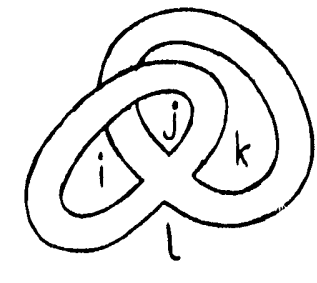

(b)

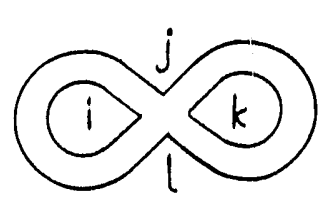

(c)

Figure 1. The three lowest-order graphs for the quartic model.

commuting $M_{i j}$ past $M_{k l}$. Symmetry factors can be calculated as in the usual matrix models, following the rules of ref. [5].

For a quartic potential

$$
V(M)=\frac{1}{2} \operatorname{str} M^{2}+g \operatorname{str} M^{4}
$$

the lowest-order contribution to the fiee onergy comes from the two-loop graphs in figure 1, which represent $\left\langle\operatorname{str} M^{4}\right\rangle$. Evaluating them gives

$$
\begin{aligned}
g\left\langle\operatorname{str} M^{4}\right\rangle=g \sum_{i, j, k, l}(-)^{i} & {\left[\left\langle M_{i j} M_{k j}^{*}\right\rangle\left\langle M_{k l} M_{i l}^{*}\right\rangle+(-)^{(j+k)(k+l)}\left\langle M_{i j} M_{l k}^{*}\right\rangle\left\langle M_{j k} M_{i l}^{*}\right\rangle\right.} \\
& \left.\quad+(-)^{(j+l)(l+i)}\left\langle M_{i j} M_{i l}^{*}\right\rangle\left\langle M_{j k} M_{l k}^{*}\right\rangle\right] \\
= & g \sum_{i, j, k, l}\left[(-)^{i+j+l} \delta_{i k} \delta_{j j} \delta_{k i} \delta_{l l}+(-)^{i+j+k}(-)^{(j+k)(k+l)} \delta_{i l} \delta_{j k} \delta_{j i} \delta_{k l}\right. \\
& \left.+(-)^{i+j+k}(-)^{(j+l)(l+i)} \delta_{i i} \delta_{j l} \delta_{j l} \delta_{k k}\right] \\
= & g\left[(m-n)^{3}+(m-n)+(m-n)^{3}\right] .
\end{aligned}
$$

The three contributions to (3.3) come respectively from the three graphs in figure 1. Graphs (a) and (c) are planar, while (b) is nonplanar. It was possible to rescale $g$ so that (3.3) is identical to the standard Hermitian matrix result[ $[j]$. If $g=\widehat{g}(m-n)^{-1}$, then (3.3) may be written

$$
\widehat{g}(m-n)^{-1}\left\langle\operatorname{str} M^{4}\right\rangle=\widehat{g}\left[2(m-n)^{2}+1\right]
$$

The power of $(m-n)^{2}$ is $(1-G)$, where $G$ is the genus of the surface identified with the graph by filling in faces in the usual manner. 


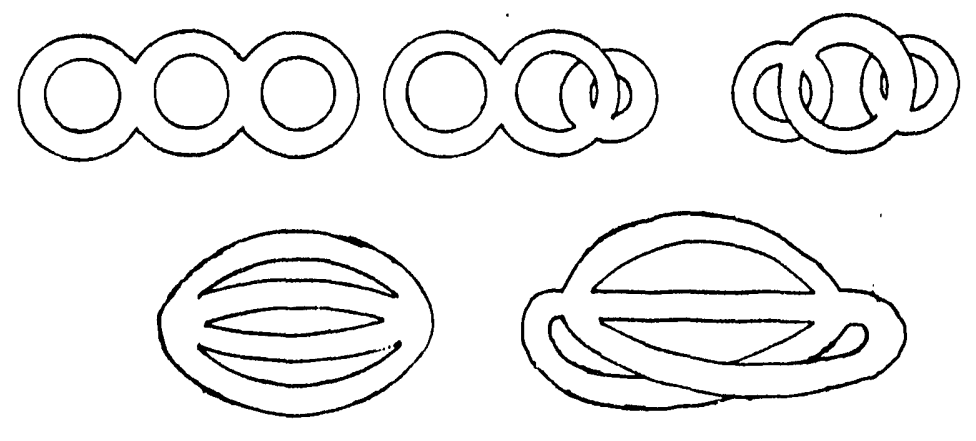

Figure 2. The inequivalent connected three-loop graphs with two quartic vertices.

This provides evidence that the supermatrix model will have a $2 d$ gravity interpretation, when $m, n \rightarrow \infty$ with $n / m$ fixed. A computation of all three-loop connected graphs containing two quartic vertices provides further evidence for this. The total contribution of these graphs, shown in figure 2, to the free energy is

$$
\frac{\widehat{g}}{2 !(m-n)^{2}}\left\langle\left(\operatorname{str} M^{4}\right)^{2}\right\rangle_{\mathrm{conn} .}=\widehat{g}\left[18(m-n)^{2}+30\right],
$$

which is identical to the ordinary Hermitian matrix result[5], with $N$ replaced by $m-n$. In fact, it is clear that in an arbitrarily complex diagram, each closed index loop (face in a generalized triangulation of the surface) will give a factor of $m \pm n$. If the pattern suggested by low order computations persists, then only $m-n$ will occur, and the model will be precisely equivalent to the $|m-n| \times|m-n|$ Hermitian matrix model. (Ref. [17] provides some further evidence that this is the case.) However, if any factors of $m+n$ occur, the topological expansion will still go through, but the weighting factors for various surfaces will be changed. More powerful combinatorial methods are needed to settle this question.

If the identification with the $|m-n|$ Hermitian model persists, then this may suggest a connection with the bosonic sector of a subcritical superstring theory. Ordinary superstrings have a bosonic Neveu-Schwarz sector which by itself is closely analogous to the purely bosonic string, in the sense that is can contain 
the same massless spacetime fields (gravity, etc.) with the same low-energy effective action. The fermions play a purely internal role in this sector. This seems analogous to the role played by the fermions in a $c$-type supermatrix. They play an internal role, but in a "spacetime" sense, the model behaves as a bosonic one. Adjoining a-type supermatrices could provide an analog of a Ramond sector, containing true fermions. Quenching, or constraining some elements of a supernatrix element, may also lead to a supersymmetric theory[16].

\section{Physical Supermatrix Model}

Having considered the case where the even entries in the supermatrix are pure numbers, it is now natural to turn to the other relatively simple case, where the eigenvalues of the supermatrix are pure real numbers. Such Hermitian supermatrices may be thought of as observables in a quantum-mechanical system defined on a supermanifold.

Incorporating the constraint that $M$ have real eigenvalues directly into (2.3) is complicated. A Jacobian factor will be needed, which is difficult to compute in general. However, working with the eigenvalue reduction (2.7) is simple in this case. The eigenvalues $\lambda_{i}$ are integrated over the ordinary real numbers, and the supergroup integral over $U(m \mid n)$ factorizes. In fact, that integral vanishes, as can be seen from (2.11) for the (1|1) supermatrix example, or in general from the fact that (2.3) vanishes when $\partial \lambda_{i} / \partial B_{\mu \alpha}=0$. In any case, the supergroup integral will be dealt with by fixing the gauge, and simply dropping it. This gives the closest analog of the ordinary matrix model eigenvalue reduction. The remaining eigenvalue partition function will be essentially a vector model whose form and symmetries are inherited from the underlying supermatrix model.

The only ingredient still needed in (2.7) is the determinant $\Delta$. This can be calculated by representing $U$ in (2.6) as $e^{A}$ with $A$ anti-Hermitian. Then, if $\Lambda$ is

\footnotetext{
$\star$ Such systems are described in chapter 5.3 of ref. [12].
} 
the diagonalized matrix,

$$
\left(U \Lambda U^{\dagger}\right)_{i j}=A_{i j}\left(\lambda_{j}-\lambda_{i}\right)+\ldots
$$

and, keeping track of the Grassmann character of the delta functions in (2.6), the determinant is found to be

$$
\Delta(\lambda)=\prod_{\mu<\nu}\left(\lambda_{\mu}-\lambda_{\nu}\right)^{2} \prod_{\alpha<\beta}\left(\lambda_{\alpha}-\lambda_{\beta}\right)^{2} \prod_{\mu, \alpha}\left(\lambda_{\mu}-\lambda_{\alpha}\right)^{-2}
$$

in the index conventions of (2.1). The poles occur at supermatrices which technically are not diagonalizable for the reasons noted in section 2 . The gav ge-fired physical supematrix model is then defined by the equation

$$
Z_{m n}(\beta)=\int \prod_{i} d \lambda_{i} \Delta(\lambda) \exp -\beta\left(\sum_{\mu=1}^{n} V\left(\lambda_{\mu}\right)-\sum_{\alpha=1}^{n} V\left(\lambda_{\alpha}\right)\right) .
$$

This model is clearly inequivalent to the usual hermitian model, due to the presence of a denominator in (4.2). The ordinary matrix models were introduced as a model for describing the eigenvalue distribution of a random physical operator. In that case, Wigner[1] observed that the eigenvalues repel each other, and Dyson[2] noted that they can be interpreted as a gas of charged particles with $e^{2}=1 / \beta$ in one dimension, at temperature $1 / \beta$. In the limit $N, \beta \rightarrow \infty$ with $\beta<N$, the gas freezes into a crystal with a charge distribution determined by the external potential $V$. The critical case takes $\beta / N \rightarrow 1$, so that fluctuations about the crystal become important, and in the perturbative expansion, higher genus graphs contribute comparably to planar ones[7-9].

The physical analog for the supermatrix model is a two-component plasma in one dimension, consisting of equal and opposite charges $\pm \beta^{-1 / 2}$ at temperature $1 / \beta$, in an external potential $V$. The $\lambda_{\mu}$ may be considered to be $m$ positive charges, while the $\lambda_{\alpha}$ are $n$ negative charges. At very low temperatures, the positive charges will pair with a negative partner, leading to an effective theory of $n$ dipoles interacting with $m-n$ positive charges when $m>n$. 
Assume $m>n$ and introduce variables

$$
x_{i}=\frac{1}{2}\left(\lambda_{1}+\lambda_{m+1}\right) . \quad u_{1}=\frac{1}{2}\left(\lambda_{1}-\lambda_{m+1}\right), \quad y_{a}=\lambda_{n+a}
$$

for $i=1, \ldots, n$, and $a=1, \ldots, m-n$. In the limit where the first $n$ positive and negative charges pair. $x_{i}$ is a center of mass coordinate for the $i$ th dipole, $u_{i}$ is its charge displacement, and $y_{s}$ labels the $m-n$ left-over positive charges. In terms of these variables. (1.3) may be rewritten

$$
\begin{aligned}
Z_{m n}=2^{n} & \int \prod_{i=1}^{n} \frac{d x_{i} d u_{i}}{u_{i}^{2}} \prod_{a=1}^{m} d y_{a} \prod_{i \neq j}\left[\frac{1-\left(u_{i}-u_{j}\right)^{2}\left(x_{i}-x_{j}\right)^{-2}}{1-\left(u_{i}+u_{j}\right)^{2}\left(x_{i}-x_{j}\right)^{-2}}\right]^{2} \\
& \times \prod_{i=a}\left[\frac{1+u_{i}\left(x_{i}-y_{a}\right)^{-1}}{1-u_{i}\left(x_{i}-y_{a}\right)^{-1}}\right]^{2} \prod_{a, b}\left(y_{a}-y_{b}\right)^{2} \\
& \times \exp -3\left\{\sum_{a} V\left(y_{a}\right)+\sum_{i}\left[V\left(x_{i}+u_{i}\right)-V\left(x_{i}-u_{i}\right)\right]\right\} .
\end{aligned}
$$

Clearly, $Z_{m n}$ is dominated by configurations where $u_{i} \rightarrow 0$, and by configurations equivalent to this up to index permutations. These configurations actually cause $Z$ to diverge, which is a price for simply ropping the $U(m \mid n)$ integral. However, once the internal energy of the dipoles is regulated by cutting off the displacements, no further divergences occur, provided the potential is well-chosen.

For simplicity, further attention will be restricted to the neutral plasma, with $m=n$. In the dipole approximation, cutting off the displacements at $\delta_{i}$ gives

$$
\begin{aligned}
Z \cong 2^{n} n ! \int_{-\infty}^{\infty} d^{n} x \int_{\delta_{1}} \frac{d u_{i}}{u_{i}^{2}} \\
\quad \times \sum_{\epsilon_{i}= \pm 1} \exp -\beta\left\{\sum_{i} \epsilon_{i}\left[V\left(x_{i}+u_{i}\right)-V\left(x_{i}-u_{i}\right)\right]+\sum_{i \neq j} \epsilon_{i} \epsilon_{j} V_{i j}\right\}
\end{aligned}
$$

where $u_{i}$ are now considered to be small and positive and the dipole interaction 
is

$$
V_{i j}==-\frac{4}{\beta} \tanh ^{-1}\left[\frac{2 u_{i} u_{j}}{\left(x_{i}-x_{j}\right)^{2}-u_{i}^{2}-u_{j}^{2}}\right] \cong-\frac{8}{\beta} \frac{u_{i} u_{j}}{\left(x_{i}-x_{j}\right)^{2}}
$$

To lowest order in $u$, the interaction is attractive, but the exact form shows that higher order repulsive effects will prevent a singularity in $V_{i j}$ for $x_{i} \rightarrow x_{j}$. The dipole interaction with the external potential is $\epsilon_{i} u_{i} V^{\prime}\left(x_{i}\right)$, where $V^{\prime}$ is the derivative of $V$ with respect to $x$. Therefore, positively polarized dipoles are drawn to the minima of $V^{\prime}$ while negative ones pre drawn to its maxima.

It is informative to choose a simple potential, and find the behavior of the free energy near a saddle point. Consider the example of a cubic potential

$$
\beta V(x)=\frac{1}{2} x^{2}+\frac{1}{3} \beta^{-1 / 2} g x^{3} .
$$

In this case, the true minima of the potential should come from unstable configurations where negative dipoles lump together and run off to infinity, or positive ones all condense at $x=-\beta^{1 / 2} g^{-1}$. The former case will be neglected, since it can be tamed by modifying the potential at large $|x|$ (a situation familiar in matrix models). The latter case is important, but requires the inclusion of the higher-order repulsive effects in $V_{i j}$ for meaningful results.

Therefore, an unstable stationary point, where negative dipoles are repelled from the minimum of $V^{\prime}$ but attracted to each other, will be considered for illustrative purposes. (This is opposite the usual situation for matrix models.) It is a simple enough case to allow a direct comparison with the saddle point analysis of the standard matrix model[4], and it is useful to know exactly how the supermatrix analysis differs.

In the $n, \beta \rightarrow \infty$ limit, it is useful to define a continuum variable $x(t)=$ $n^{-1 / 2} x_{\text {: }}$ with $t=i / n$. Near the saddle point, the dipole approximation should be 
valid, and we will work with the effective dipole energy

$$
n^{2} \beta E=2 \beta \int_{0}^{1} d t p(t) V^{\prime}(x(t))-8 \int_{0}^{1} \int_{0}^{1} d t d t^{\prime} \frac{p(t) p\left(t^{\prime}\right)}{\left(x(t)-x\left(t^{\prime}\right)\right)^{2}}+\frac{2}{n} \int_{0}^{1} d t \log \left(n^{1 / 2} p(t)\right)
$$

The continuum dipole is defined to be $p(t)=n^{-1 / 2} \epsilon_{i} \delta_{i}$ in terms of the polarization and cutoff in (4.6), and for the cubic potential,

$$
\beta V^{\prime}(x(t))=x(t)+\widehat{g} x^{2}(t), \quad \widehat{g}=\left(\frac{n}{\beta}\right)^{1 / 2} g .
$$

Introducing a dipole density $P(x)=p(t) d t / d x$ and dropping the dipole selfenergy term gives

$$
n^{2} \beta E=2 \int d x P(x)\left(x+\widehat{g} x^{2}\right)-8 \iint d x d x^{\prime} \frac{P(x) P\left(x^{\prime}\right)}{\left(x-x^{\prime}\right)^{2}}
$$

The saddle point equation $\delta E / \delta p(t)=0$ is satisfied when $\partial_{x}(\delta E / \delta P(x))=0$. For (4.11) this implies

$$
x+\frac{1}{2 \widehat{g}}=-\frac{16}{\widehat{g}} f d x^{\prime} \frac{P\left(x^{\prime}\right)}{\left(x-x^{\prime}\right)^{3}}
$$

using the same principle part prescription as ref. [4] to handle the coincident point. (Recall that (4.6) shows that the coincident limit is not truly singular.)

Since the saddle point which will be found is the unstable one with negative dipoles clustered about the minimum of $V^{\prime}$ (using some foresight), it is convenient to define the integrated dipole density as

$$
\int d x P(x)=-p_{0}
$$

The quantity $p_{0}$ is determined by how $n^{-1 / 2} \delta_{i}$ is tuned as $n \rightarrow \infty, \delta_{i} \rightarrow 0$, and should be thought of as a new parameter of the model. It is also convenient to 
introduce a new variable

$$
w=\imath\left(x+\frac{1}{2 \widehat{g}}\right)
$$

centered at the minimum of $V^{\prime}$, with scale $\kappa^{-4}=32 p_{0} / \widehat{g}$ chosen for later simp.icity. Then the normalized negative dipole density $\rho$ defined by

$$
P(x)=-\frac{1}{2} \pi \kappa p_{0} \rho(w)
$$

has total integral $+2 / \pi$. (This integral was fixed for later convenience.)

In the new variables, the stationary condition (4.12) becomes

$$
w=\frac{\pi}{4} f d w^{\prime} \frac{\rho\left(w^{\prime}\right)}{\left(w-w^{\prime}\right)^{3}}, \quad|w|<a
$$

Due to the symmetry of the problem, $\rho(w)$ will be an even function, with support in an interval $[-a, a]$ to be determined. The density does not have to be positive, since dipoles can have two polarizations, but the integral must be $2 / \pi$ by construction. A solution can be found following ref. [4]'s treatment of the ordinary quartic matrix model, by introducing a function

$$
F(w)=\frac{\pi}{4} \int_{-a}^{a} d w^{\prime} \frac{\rho\left(w^{\prime}\right)}{\left(w-w^{\prime}\right)^{3}}
$$

which is analytic for complex $w$ with cut $[-a, a]$, behaves as $\frac{1}{2} w^{-3}$ for $|w| \rightarrow \infty$, and satisfies

$$
F(w \pm i \epsilon)=w \mp \frac{1}{2} \pi i \rho^{\prime \prime}(w)
$$

for $w$ in $[-a, a]$. 
The solution is

$$
F(w)=w-\sqrt{w^{2}-w^{-2}}
$$

which requires $a=1$ and

$$
\rho^{\prime \prime}(w)=\frac{2}{|w|} \sqrt{1-w^{4}}
$$

for $|w|<1$. Integrating once gives

$$
\rho^{\prime}(w)=\left(\sqrt{1-w^{4}}-\tanh ^{-1} \sqrt{1-w^{4}}\right) \operatorname{sgn} w
$$

The logarithmic singularity at $w=0$ is integrable, and the resulting density will be finite. The integration constants are fixed by adding a term

$$
-h(1-|w|)
$$

to $\rho(w)$, with coefficient $h$ chosen such that the integrated density is $2 / \pi$, as required by the defining conditions. Eqn. (4.21) may be integrated numerically, leading to $h \cong .147$, for which the final solution $\rho(w)$ is shown in figure 3 .

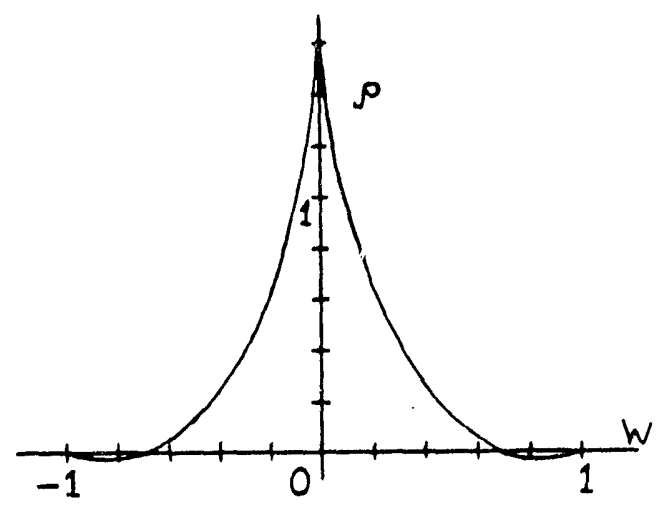

Figure 3. The normalized negative dipole density $\rho(w)$ as a function of the coordinate $w$ centered at the minimum of the quadratic potential $V^{\prime}$. 
The energy for this configuration is found by substitutizg $\rho$ into (4.11). Using some integration by parts, (4.20), (4.21), and (4.22) suffice to obtain $\beta E$. The double integral can be done with the help of (4.12) as in ref. [4], leading to

$$
n^{2} \beta E=\frac{3}{8} \widehat{g}^{-1} p_{0}+\frac{1}{3} \pi \widehat{g}^{1 / 2} p_{0}^{3 / 2}\left\{I(\alpha)-\frac{1}{3}+h \log (1-\alpha)\right\}
$$

where $\alpha^{2}=2^{9} \hat{g}^{3} p_{0}$ and

$$
I(\alpha)=-\int_{0}^{1} \frac{d z}{z} \sqrt{1-z^{2}} \log (1-\alpha z)=\frac{\sqrt{\pi}}{2} \sum_{n=1}^{\infty} \frac{\Gamma\left(\frac{n}{2}\right)}{\Gamma\left(\frac{n+1}{2}\right)} \frac{\alpha^{n}}{n(n+1)}
$$

The surface interpretation of a matrix model depends only on the non-analytic behavior of the partition function. The expression (4.23) has non-analytic behavior when

$$
2^{3} g^{3} p_{0}\left(\frac{n}{\beta}\right)^{3 / 2} \longrightarrow 1
$$

This can occur for $n / \beta \rightarrow 1$ if $g^{3} p_{0}=2^{-9}$.

In fact, it appears unlikely that this stationary point can have much relevence to a $2 d$ gravity model. Recalling that $\rho$ is the density of negative dipoles, and that negative dipoles want to climb the potential, it is clear that this is actually an unstable stationary noint, so it is uncertain whether it of any physical use. Certainly the energy (4.23) of this configuration will not be a good approximation to the free energy. (However, inverted potentials sometimes come up in scaling limits of matrix models[9], so instabilities are not always as bad as they seem.) This is the only kind of stationary point that can be found within the limits of the dipole approximation of the one-dimensional plasma, however. A more exact treatment would be desirable, but this is more difficult than for the usual matrix models, where the orthogonal polynomial method yields relatively easy exact results. 


\section{Conclusions}

The motivation for introducing supermatrix models is their apparent mathematical similarity to ordinary matrix models. This suggests that they could provide a way to incorporate fermions in a matrix model without sacrificing integrability.

However, when these models are investigated in detail, it becomes clear that the nature of superspace gives them very different properties from ordinary matrix models, and that certain useful tools, especially the orthogonal polynomial method, are not easily applied. The source of the most unique features of supermatrix models is the inherent ambiguity in integrating over even Grassmann variables.

The most obvious definition of the supermatrix integral gives a perturbative expansion which implies that a scaling limit of the model will have a $2 d$ gravity interpretation (but not supergravity, since the models considered have only bosonic coupling constants). The fact that the superunitary integral does not decouple in this case means that the eigenvalue reduction an orthogonal polynomial method are not reedily applicable, although eventually suitable extensions may be found.

The leading terms in the perturbative expansion of the quartic $(m \mid n)$ Hermitian supermatrix model are identical to those of the $(m-n)$ bosonic Hermitian matrix model. This may suggest a connection to the "spacetime" bosonic sector of a subcritical superstring, to which fermionic partners must be adjoined to complete the theory. The fact that supermatrices contain odd Grassmann variables is not in itself reason to believe that they would constitute a supersymmetric theory by themself. Perhaps the appropriate partners are supermatrices with the bosonic and fermionic entries enterchanged.

Since the supermatrix integrand depends only on invariants, it will actually vanish whenever the superunitary integral does decouple. Nevertheless, it is 
useful tu define such models by gauge-fixing, since they provide the closest superanalog of Wigner's model for the eigenvalue distribution of a physical operator. The result is that the eigenvalues of a physical super-operator separate into two classes. Within each class, the eigenvalues repel, as in Wigner's model. However, eigenvalues in each class attract those of the othe1. If any real system has such properties, perhaps supermatrices could provide a useful model for some of its statistical properties, as matrix models did with some degree of success[18] for the highly-excited states of nuclei.

In analogy with the Dyson gas interpretation of matrix models, the eigenvalues of a supermatrix may be though of in term: of a two-component plasma in one dimension. This physical analog could provide useful intuition for solving the matrix model. Conversely, the matrix model, when ufficiently developed, could provide a new source of information about such plasmas. Electron-hole plasmas in optically-excited semiconductors are a well-studied system[19] which may be somewhat analogous.

The analysis presented here should be considered preliminary. Acsuming that opposite charges condense into pairs at low temperatures, an effective dipole gas was constructed, and one of the stationary points for a cubic potential was analyzed. Unfortunately, the only stationary point found within the dipole approximation is unstable, so its physical applicability is questionable. For example, it may be helpful to include effective neutral particles containing more than two charges. Developing a more exact treatment of the plasma could reveal some interesting physics, and perhaps new $2 d$ gravity models.

\section{Acknowledgements:}

I would like to thank M. Awada, Z. Qiu, P. Ramond, S.-J. Rey, S.-J. Sin, and R.P. Woodard for discussions and comments. 


\section{REFERENCES}

1. E. P. Wigner, Ann. Sath. 62 (1955) 548; 65 (1957) 203

2. F.J. Dyson, J. Math. Phys. 3 (1962) 140, 157, 166

3. M.L. Mehta, Random Matrices (Academic Press, 1967)

4. E. Brézin, C. Itzỵkson, G. Parisi and J.B. Zuber, Commun. Math. Phys. 59 (197s) 35

5. D. Bessis. C. Itzykson and J.B. Zuber, Adv. Appl. Math. 1 (1980) 109

6. R.C. Penner, J. Diff. Geom. 27 (1988) 35

T. E. Brezin and V.A. Kazakov, Phys. Lett. B236 (1990) 144

8. M.R. Douglas and S.H. Shenker, Nucl. Phys. B335 (1990) 635

9. D.J. Gross and A.A. Migdal, Phys. Rev. Lett. 64 (1990) 127; Nucl. Phys. B340 (1990) 333

10. V. Periwal and D. Shevitz, Phys. Rev. Lett. 64 (1990) 1326; Nucl. Phys. B344 (1990) 731

11. J. Distler and C. Vafa, Mod. Phys. Lett. A5 (1990) 2135

12. B. DeWitt, Supermanifolds (Cambridge University Press, 1984)

13. M. Douglas, Phys. Lett. 238B (1990);

E.J. Martinec, U. Chicago preprint EFI-90-62;

A. Gerasimov, A. Markashov, A. Mironov, A. Morozov and A. Orlov, Moscow preprint Print-90-0576

14. P. Difrancesco, J. Distler and D. Kutasov, Mod. Phys. Lett. A5 (1990) 2135;

M. Awada, U. Florida preprints UFIFT-HEP-90-18 and -29

15. C. Vaz, U. Cincinnati preprint UCTP-102-91

16. G. Gilbert and M.J. Perry, U. Maryland preprint UMDEPP 91-211 
17. L. Alvarez-Gaumé and J.L. Manẽs, CERN preprint CERN-TH. 6067/91

18. J.L. Rosen, J.S. Desjardins, J. Rainwater and W.W. Havens, Jr., Phys. Rev. 118 (1960) 687

19. C. Klingshirn and H. Haug, Phys. Rep. 70 (1981) 315

\section{DISCLAIMER}

This report was prepared as a 7 account of work sponsored by an agency of the United States This report was prepared Unit a States Government nor any agency thereof, nor any of their Government. Neither the Unit id States Government nor any agences legal liability or responsiemployees, makes any warranty, express or implied, of any information, apparatus, product, or

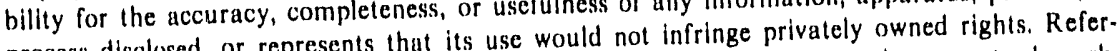
process disclosed, or represents that its use would not infringe privately owned rights. Reforader ence herein to any specific commercial product, process, or service by trade nase, lraderem. manufacturer, or otherwise does not necessarily constitute or imply its endorseof. The views mendaiton, or favoring by the United States Government or any agency thereof. The views and opinions of authors expressed herein do nof 

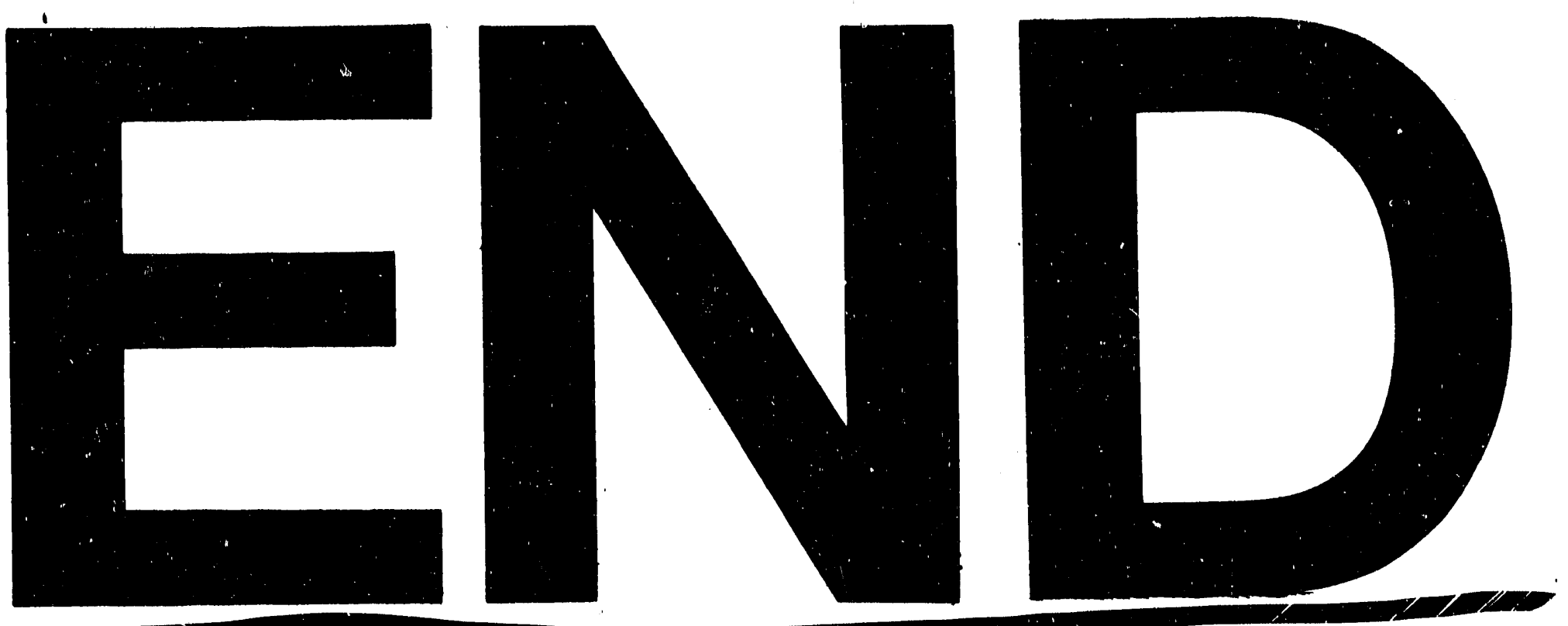

.
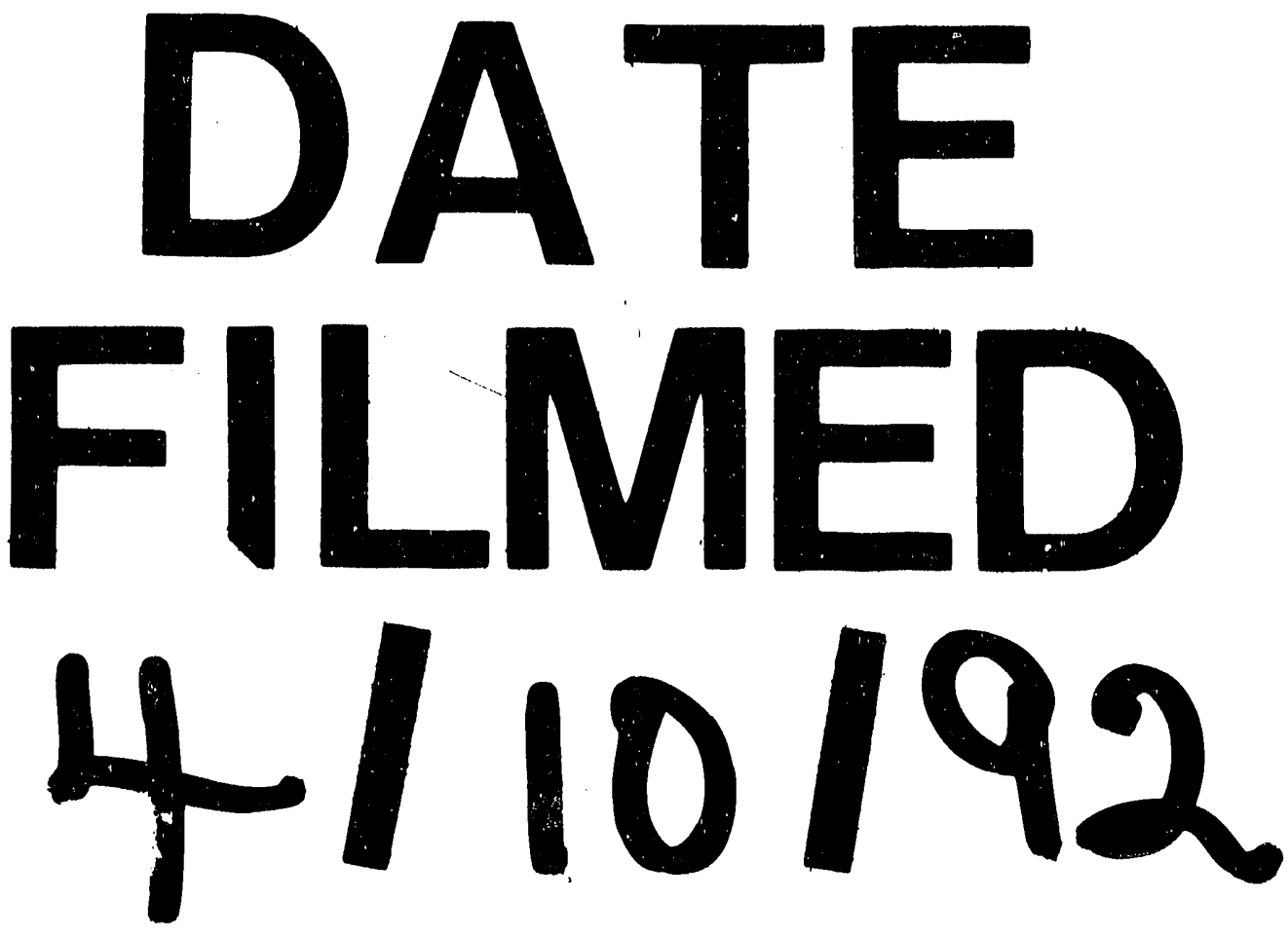


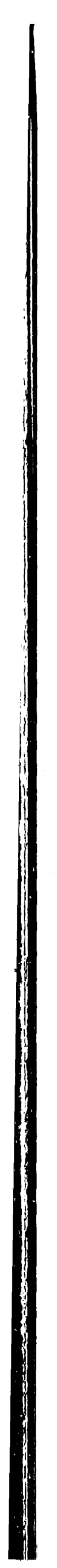

